\title{
Diffraction-limited polarimetric imaging of protoplanetary disks and mass-loss shells with VAMPIRES
}

Norris, Barnaby R., Tuthill, Peter, Jovanovic, Nemanja, Lozi, Julien, Guyon, Olivier, et al.

Barnaby R. M. Norris, Peter Tuthill, Nemanja Jovanovic, Julien Lozi, Olivier Guyon, Nick Cvetojevic, Frantz Martinache, "Diffraction-limited polarimetric imaging of protoplanetary disks and mass-loss shells with VAMPIRES," Proc. SPIE 11203, Advances in Optical Astronomical Instrumentation 2019, 112030S (3 January 2020); doi: 10.1117/12.2539998 


\title{
Diffraction-limited polarimetric imaging of protoplanetary disks and mass-loss shells with VAMPIRES
}

\author{
Barnaby R. M. Norris ${ }^{\mathrm{a}, \mathrm{b}}$, Peter Tuthilla, Nemanja Jovanovic ${ }^{\mathrm{c}}$, Julien Lozi ${ }^{\mathrm{d}}$, Olivier Guyon ${ }^{\mathrm{d}}$, \\ Nick Cvetojevic ${ }^{\mathrm{e}}$, and Frantz Martinache ${ }^{\mathrm{e}}$

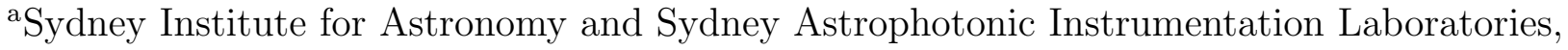 \\ School of Physics, University of Sydney, NSW 2006, Australia \\ ${ }^{\mathrm{b}}$ Australian Astronomical Observatory, Faculty of Science and Engineering, Macquarie \\ University, NSW 2109, Australia \\ ${ }^{\mathrm{c}}$ Department of Astronomy, California Institute of Technology, 1200 E California Blvd, \\ Pasadena, CA 91125, USA \\ ${ }^{d}$ National Astronomical Observatory of Japan, Subaru Telescope, 650 N. A'Ohoku Place, Hilo, \\ Hawaii 96720, U.S.A \\ ${ }^{\text {e}}$ Laboratoire Lagrange, Universite Cote d'Azur, Observatoire de la Cote d'Azur, CNRS, Parc \\ Valrose, Bat. H. FIZEAU, 06108 Nice, France
}

Keywords: Interferometry, polarimetry, exoplanets, aperture masking, Subaru telescope, protoplanetary disks

\section{INTRODUCTION}

Both the birth and death of a stellar system are areas of key scientific importance. Whether it's understanding the process of planetary formation in a star's early years, or uncovering the cause of the enormous mass-loss that takes place during a star's dying moments, a key to scientific understanding lies in the inner few AU of the circumstellar environment. Corresponding to scales of 10s of milli-arcseconds, these observations pose a huge technical challenge due to the high angular-resolutions and contrasts required. A major stumbling block is the problem of the Earth's own atmospheric turbulence. The other difficulty is that precise calibration is required to combat the extremely high contrast ratios and high resolutions faced. By taking advantage of the fact that starlight scattered by dust in the circumstellar region is polarized, differential polarimetry can help achieve this calibration. Spectral features can also be utilized.

\section{THE VAMPIRES INSTRUMENT}

VAMPIRES (Visible Aperture Masking Polarimetric Interferometer for Resolving Exoplanetary Signatures) is a recently commissioned and newly upgraded high-angular resolution imager which capitalizes on these techniques. Developed by the University of Sydney in conjunction with the SCExAO extreme adaptive-optics project, it is currently deployed on the Subaru telescope as part of the SCExAO system. It leverages both sparse-aperturemasking interferometry and high-speed speckle imaging to beat the seeing and obtain super-diffraction-limited imaging, and simultaneous polarized-differential imaging and/or spectral-differential imaging to beat the contrast problem.

In contrast to conventional coronagraphic techniques, aperture masking interferometry has demonstrated the ability to image faint companions at resolutions well beyond the diffraction limit. VAMPIRES combines this with polarimetry to directly image structure in the inner-most regions of protoplanetary systems and evolved stars, at visible wavelengths. VAMPIRES uses starlight scattered by dust in such regions to precisely image the disks, shell, gaps, knots and waves that are key to understanding disk evolution and planet formation. It also images the inner mass-loss dust-shells of evolved stars, directly resolving the dust condensation region and the root of asymmetries. Furthermore, it can use spectral features - such as the $\mathrm{H} \alpha$ emission line from an accreting planetismial or stellar pulsation shock - to perform differential measurements.

Further author information: (Send correspondence to B. R. M. N.)

B. R. M. N.: E-mail: barnaby.norris@sydney.edu.au

Advances in Optical Astronomical Instrumentation 2019, edited by Simon Ellis, Céline d'Orgeville, Proc. of SPIE Vol. 11203, 112030S · @ 2020 SPIE · CCC code: 0277-786X/20/\$21 · doi: 10.1117/12.2539998 
With a spatial resolution of 10 mas and with a maximum field of view of 500 mas, VAMPIRES perfectly compliments coronagraphic observations in the near-IR, and in fact can operate simultaneously with IR coronagraphic observations by utilizing the otherwise unused visible wavelengths. High resolutions and dynamic ranges are enabled by VAMPIRES' recently-upgraded triple-layered polarimetric differential calibration system, using simultaneous polarized channel splitting, fast ferroelectric-liquid-crystal channel modulation and half-wave-plate based channel switching. Multiple opto-mechanical systems allow various aperture-masks, polarization devices and imaging subsystems to be deployed, with the final interferogram imaged at high cadence using a pair of EMCCD detectors.

Now in regular operation at the Subaru telescope, VAMPIRES has demonstrated polarized differential visibility precisions of 1 part in 1000, and differential closure phases of a fraction of a degree. Recent science results include detections of mass-loss shells at scales of order the stellar radius, imaging of protoplanetary disk structure and direct imaging of stellar morphologies. An instrument description as well as the latest on-sky performance and science results will be presented.
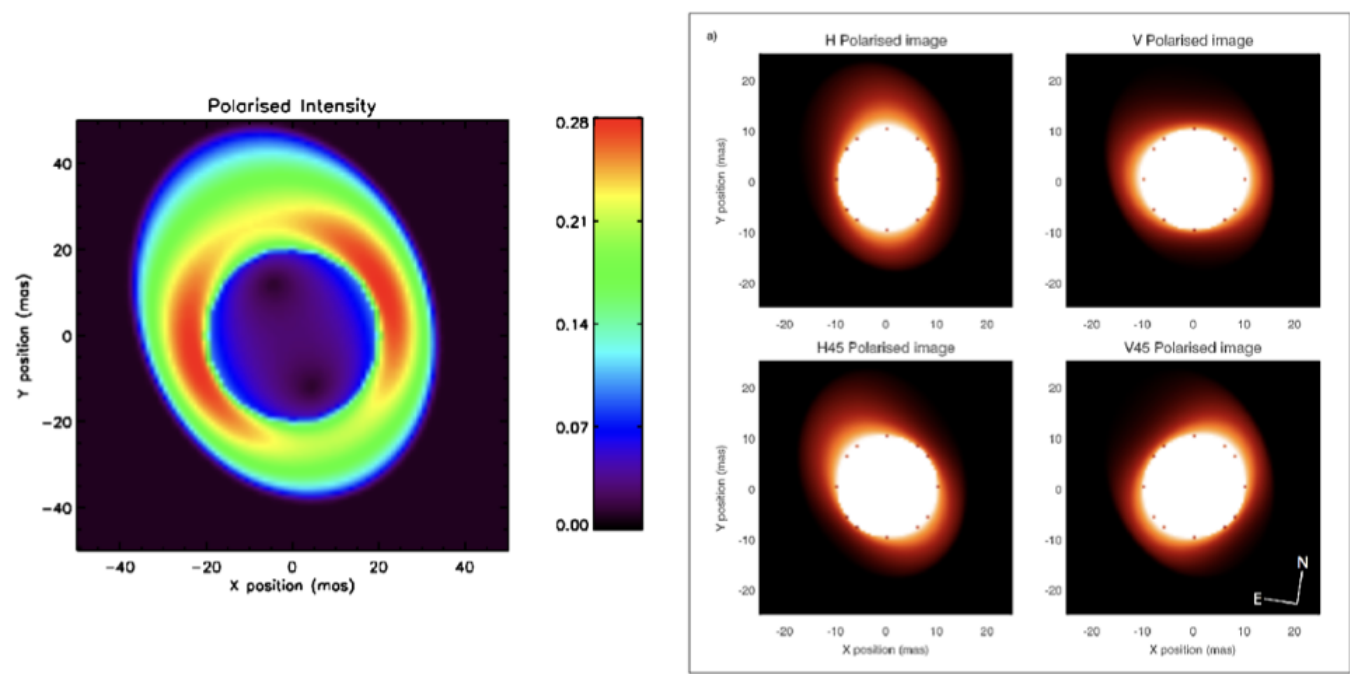

Figure 1: Model-fitting to VAMPIRES observations of the red giant star star $\mu$ Cep reveals extended, asymmetric dust shell, originating within the outer stellar atmosphere, without a visible cavity. Such low-altitude dust (likely Al2O3) important for unexplained extension of RSG atmospheres. An inner shell radius of $9.3 \pm 0.2$ milli-arcseconds is resolved.

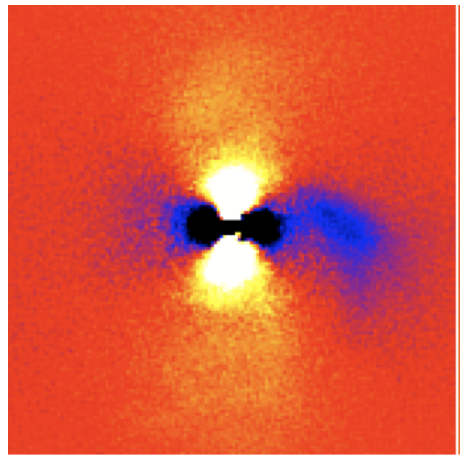

Stokes Q

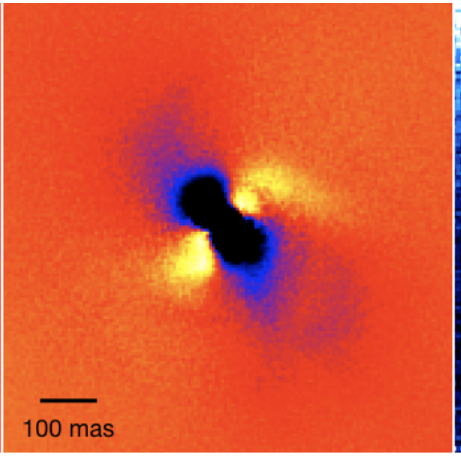

Stokes U

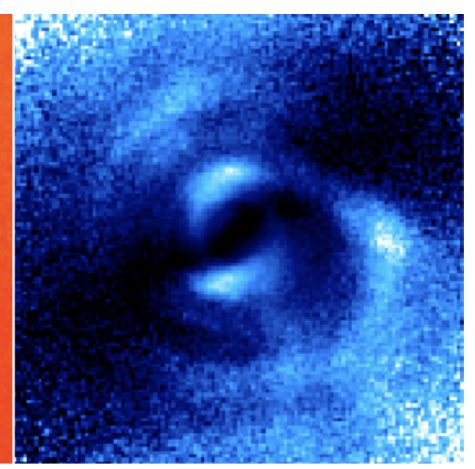

fractional $P$

Figure 2: Polarisation differential imaging of the protoplanetary disk AB Aur using VAMPIRES, shown in linear Stokes images and fractional polarisation. The extended disk plus the inner disk at 85 milli-arcseconds is seen. 